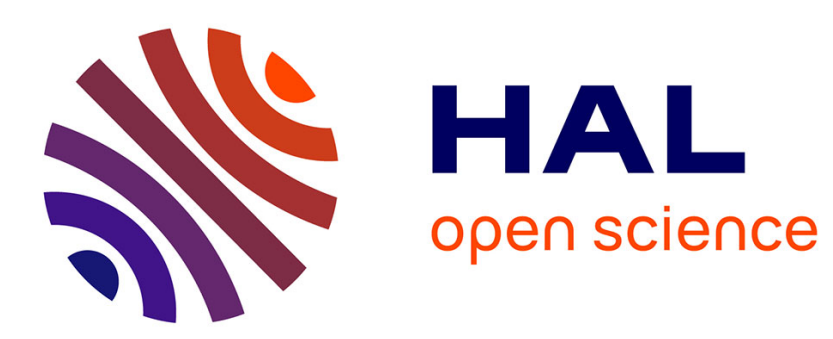

\title{
Characterization of Efficiency Enhancement in Microchannel Plate Detectors
}

S. Sijbrandij, A. Cerezo, B. Deconihout, T. Godfrey, G. Smith

\section{To cite this version:}

S. Sijbrandij, A. Cerezo, B. Deconihout, T. Godfrey, G. Smith. Characterization of Efficiency Enhancement in Microchannel Plate Detectors. Journal de Physique IV Proceedings, 1996, 06 (C5), pp.C5-297-C5-302. 10.1051/jp4:1996548 . jpa-00254427

\section{HAL Id: jpa-00254427 https://hal.science/jpa-00254427}

Submitted on 1 Jan 1996

HAL is a multi-disciplinary open access archive for the deposit and dissemination of scientific research documents, whether they are published or not. The documents may come from teaching and research institutions in France or abroad, or from public or private research centers.
L'archive ouverte pluridisciplinaire HAL, est destinée au dépôt et à la diffusion de documents scientifiques de niveau recherche, publiés ou non, émanant des établissements d'enseignement et de recherche français ou étrangers, des laboratoires publics ou privés. 
JOURNAL DE PHYSIQUE IV

Colloque C5, supplément au Journal de Physique III, Volume 6, septembre 1996

\title{
Characterization of Efficiency Enhancement in Microchannel Plate Detectors
}

\author{
S.J. Sijbrandij, A. Cerezo, B. Deconihout*, T.J. Godfrey and G.D.W. Smith \\ Department of Materials, University of Oxford, Parks Road, Oxford OX1 3PH, U.K. \\ * Laboratoire de Microscopie Ionique, Faculté des Sciences, URA 808 du CNRS, Université de Rouen, \\ Place Emile Blondel, 76821 Mont-Saint-Aignan cedex, France
}

\begin{abstract}
The detection efficiency of a channel-plate detector is mainly determined by the open area ratio of its input face, which is typically $60 \%-70 \%$. It is known that the efficiency can be enhanced by applying an electric field normal to the channel-plate surface, such that secondary electrons generated when ions strike the channel-plate surface are returned to the detector. This paper characterises the enhancement observed in channel-plate detectors for atom probe and 3-dimensional atom probe applications. In a double channel-plate detector, it was found that improvement in efficiency was approximately $30 \%$ as compared to the situation where all secondary events are lost. The secondary electron events are found to have a broader pulse height distribution, with a mean which is a factor of three lower than that of the primary ion events. Using the variation of efficiency with grid voltage, the maximum secondary electron energy was estimated to be $10 \mathrm{eV}$. This value was used to calculate the loss in time and spatial resolution which would result from the detection of secondary electrons. These effects are shown to be acceptably small within a 3-dimensional atom probe detector design, for a wide range of bias voltages. Previous work has suggested that the efficiency gain from a biassed grid drops off at grid fields in the range $25-100 \mathrm{~V} / \mathrm{mm}$. This effect is shown to have been generated by field fringing effects.
\end{abstract}

\section{INTRODUCTION}

Most atom probe instruments use micro-channelplates (MCPs) [1] as the amplification device in their single ion sensitivity detectors. Since the size of features studied with an atom probe is in the order of a few nanometers, very few atoms are available for analysis. It is therefore crucial that detection efficiency is maximised, in order to improve the statistical precision of composition measurements.

The front face of an MCP detector consists of a hexagonal array of open channels, occupying typically $60 \%-70 \%$ of the surface area. Under normal circumstances, it is assumed that only the ions entering the channels will generate an event, and therefore approximately $30 \%-40 \%$ of the ions are lost from the analysis. However, ions striking the front face of the channelplate (usually coated with chromium or nickel-chromium) can also generate small numbers of secondary electrons. The efficiency of a channel plate can therefore be enhanced by applying an electric field normal to the MCP input surface, such as to force at least some of these secondary electrons to enter one of the channels [2-5]. This is usually achieved by placing a negatively-biassed high-transmission grid a small distance in front of the front face of the MCP. Although there is a small loss in transmission because of the presence of the grid, the recovery of the secondary electrons generated when ions strike the interchannel web leads to an overall improvement in the detection efficiency. Panitz and Foesch suggested that it was sufficient to positively bias the front face of the detector with respect to its surroundings by about $24 \mathrm{~V}$ [2]. However, in their electrostatic modelling and electron trajectory calculations, they imposed a condition of an earthed equipotential at a very small

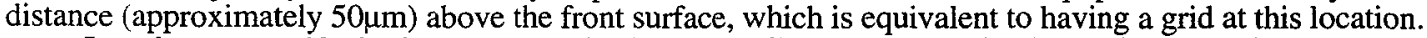

In order to quantify the improvement in detection efficiency which is obtained when using a biassed grid in an atom probe detector, Deconihout et al. designed a detector which is only half covered by the grid, and which has separate anodes so that the detection rate from the two halves of the detector can be compared directly [4]. They observed an increase in the detection efficiency for grid bias voltages below $50 \mathrm{~V}$, up to a maximum (assuming an open area ratio of $60 \%$ ) of almost $90 \%$. However at larger grid bias, the efficiency was reduced so that for a bias of $200 \mathrm{~V}(100 \mathrm{~V} / \mathrm{mm}$ field) the detection efficiency was only just over $70 \%$. 
Events resulting from the detection of a secondary electron will differ in many respects from those generated by a primary ion. Detection efficiencies for low energy electrons are in general much lower than for high energy ions, so the extent of the improvement in efficiencies obtained using a biassed grid is likely to depend on the saturation of the MCP. The secondary electron will be detected at a different place on the detector to the incident ion, and at some later time. In the context of a three-dimensional atom probe (3DAP) detector, these time and position differences must be small if they are not to lead to losses in mass and spatial resolution of the instrument.

\section{EFFICIENCY CHARACTERISATION}

A grid was incorporated into the detector of the Oxford optical position-sensitive atom probe (OPoSAP) in order to increase the overall detection efficiency of the instrument. The detector consists of a double channel plate assembly, mounted in a chevron configuration, and a P47 phosphor screen. Individual channel plates (Phillips G12-100x100-DT) are $100 \mathrm{~mm} \times 100 \mathrm{~mm} \times 1 \mathrm{~mm}$ with $12 \mu \mathrm{m}$ channels (80:1 lengthto-diameter ratio, $8^{\circ}$ bias) and nichrome coated. The active area of the detector is $90 \mathrm{~mm} \times 90 \mathrm{~mm}$. A $98 \%$ transparent nickel grid with a pitch of $2 \mathrm{~mm}$ and a wire thickness of $20 \mu \mathrm{m}$ is mounted $2.5 \mathrm{~mm}$ in front of the input face to the channel plates.

Figure 1 shows the pulse height distributions (PHD) for the detector system with $10 \mathrm{keV}$ incident $\mathrm{Ne}^{+}$ ions, when the grid was biassed at either $+200 \mathrm{~V}$ or $-200 \mathrm{~V}$ and the detector voltage was set to $1100 \mathrm{~V}$ per plate. The detection rate in this experiment was $2500-3500 \mathrm{cps}$, so that the detector was operating in the 'single pulse' mode. Charge measurement were made using an arrangement identical to a single channel of the positioning electronics in the position-sensitive atom probe (PoSAP) [6]. With a positive bias to the grid, the secondary electrons generated when ions strike the interchannel web were drawn away from the MCP, resulting in a PHD due solely to single ion events. This displays the typical form for a detector of this type, with a saturation of $75 \%$ full-width at half-maximum (FWHM). Biassing the grid negatively causes a broadening of the PHD towards low charges, due to the detection of secondary electrons. This clearly shows that the gain of the channel plates for the low energy secondary electrons is significantly less that for the primary ions. By subtracting the distribution due to ion events alone (suitably normalised), the PHD for the secondary electrons can be determined.

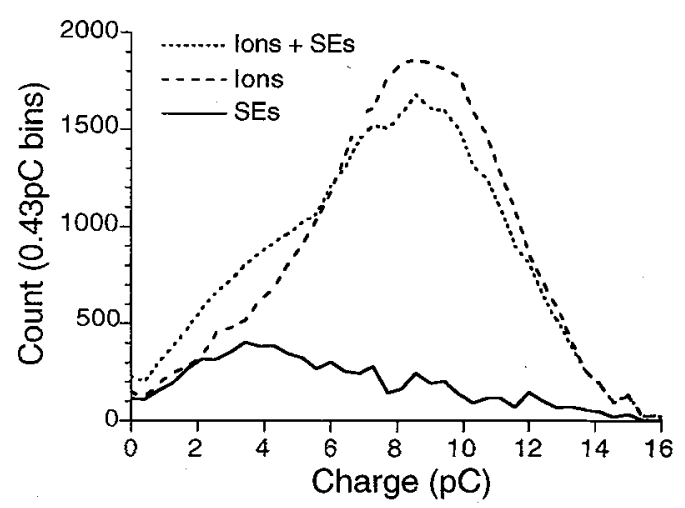

Figure 1: Pulse height distributions from the OPOSAP detector with the grid over the detector biassed to $+200 \mathrm{~V}$ (PHD due to ions alone) and $-200 \mathrm{~V}$ (distribution from both ions and secondary electrons). By subtracting the first distribution (suitably scaled) from the second, the distribution due to the secondary electrons (SEs) is obtained.

The modal charge of the secondary electron PHD is approximately one third that for the primary ion events, and is similar to the value for distributions generated by detection of single UV photons. However the long tail at high charges observed here for secondary electrons is uncharacteristic, and is probably due to the detection of multiple secondary electrons following a single ion impact on the front face of the MCP. From measurements of secondary electron yield resulting from ion impacts at different energies [7], a single $10 \mathrm{keV} \mathrm{Ne}^{2+}$ ion may generate 6-7 electrons. Even with the $60 \%$ open area ratio of the detector and the poor detection efficiency to low energy electrons, a reasonable fraction of ions generate multiple secondary electron events The lower gain of the channel plates to the secondary electrons means that the extent to which these electrons are detected depends critically on threshold setting of the discriminator used in the detection system. To maximise the gain in detection efficiency, the threshold must be set lower than would normally be the case for detecting ions alone. However this will depend on the exact design of the detector. In the curved-channel MCP used by Deconihout et al, the PHDs broadened toward higher charges when secondary electrons were detected as well as ions [4]. Gain for single electrons was obviously higher in 
this case, as was the saturation, and the detection of multiple secondary electrons from a single ion impact would generate a distribution biassed towards high charges.

The detection efficiency was characterised as a function of grid voltage by measuring the count rate as a function of grid-voltage, for events originating from a stable source of field ionised gas ions. For this purpose a tungsten specimen was imaged in neon at a temperature of $80 \mathrm{~K}$ and a voltage of $10 \mathrm{kV}$. This was found to produce a stable and uniform flux of ions onto the detector. In order to maximise the number of secondary electrons which were recovered, the threshold used for this experiment was set at a low level, equivalent to $0.3 \mathrm{pC}$, which led to an increase in the backgound noise level to a rate of approximately $100 \mathrm{cps}$. Taking this noise level into account, the primary ion rate of $1840 \mathrm{cps}$ is increased to a detection rate of $2290 \mathrm{cps}$ by the biassing of the grid. If the open area ratio of the detector is taken to be $65 \%$, then the use of a biassed grid has increased this detection efficiency to $81 \%$, which is a significant improvement. This is less then the maximum detection efficiency quoted by Deconihout et al. [4], though similar to that seen by Funsten $e t$ al. [5]. Again, the higher saturation achieved by the curved-channel MCPs allows more of the secondary electrons to be detected, leading to a higher overall efficiency.
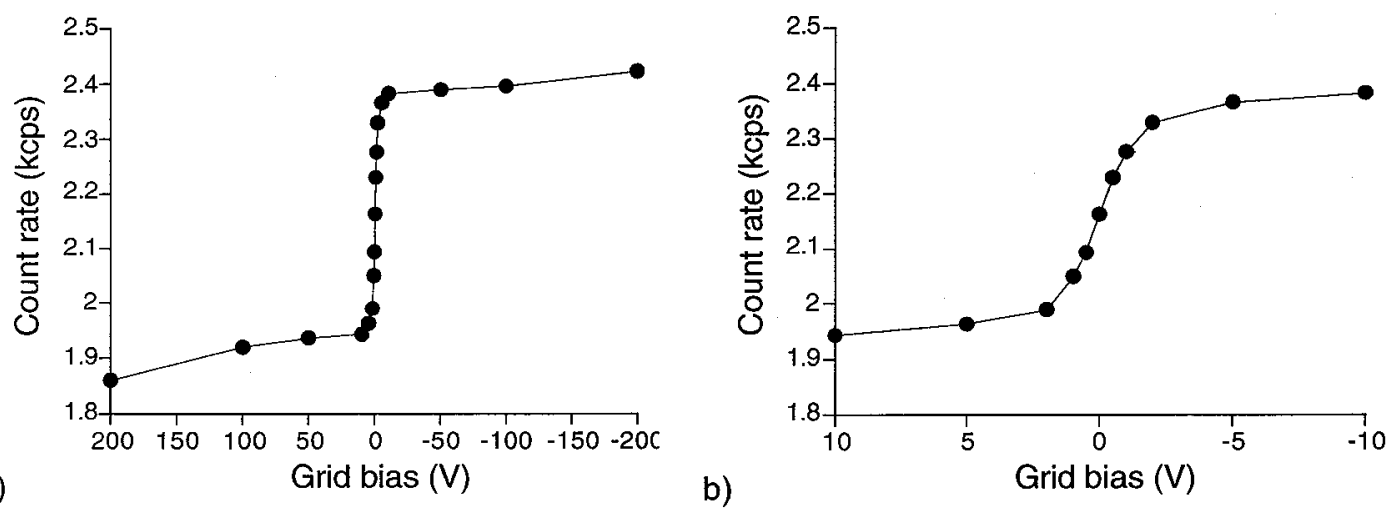

Figure 2. Plot of the ion rate as a function of grid voltage: (a) over the range +200 to $-200 \mathrm{~V}$ grid voltage and (b) over the range +10 to $-10 \mathrm{~V}$. Note that the noise level during these measurements was approximately $100 \mathrm{cps}$, and has not been subtracted from the data shown in the graphs.

\section{EFFECT ON TIMING AND SPATIAL RESOLUTION}

We can use the measurement of the effect of grid bias on detection efficiency to make an estimate of the energies of the secondary electrons generated by the impact of the ion on the front face of the MCP. Most of the improvement in detection efficiency is achieved with a bias of only $-2 \mathrm{~V}$, and almost no further improvement is seen after $-5 \mathrm{~V}$. In general, energy distributions of secondary electrons from impacts of 5$15 \mathrm{keV}$ ions show a peak at $2 \mathrm{eV}$ with a tail extending up to $10 \mathrm{eV}$ or more [7]. Measurements of the angular dependence of secondary electron yield show a $\cos \theta$ variation at these energies, where $\theta$ is the emission angle of the electron with respect to the surface normal [8]. Taking phase space considerations into account, the probability of electron emission varies as $\sin 2 \theta$, which has a peak at $\theta=45^{\circ}$. Thus, the retarding potential required to stop secondary electrons is about half the secondary electron energy, which agrees well with our observations.

The timing resolution obtained using the biassed grid may be affected by the time taken for the secondary electrons to return to the input face of the MCP. Given a field between MCP and grid of $F=V / d$, an electron emitted from the front face with energy $E$ at an angle of $45^{\circ}$ will take a time $t$ to return to the surface, given by:

$$
t=2 \frac{m}{e F} \sqrt{\frac{E}{m}}=\frac{2 d}{e V} \sqrt{m E}
$$

The worst degradation in timing resolution will occur for low grid bias and high electron energies, as might be expected. In the case of $E=10 \mathrm{eV}$ and $V=10 \mathrm{~V}, t=3 \mathrm{~ns}$ and so will contribute to a degradation in the time resolution of the detector. For $V=200 \mathrm{~V}, t=150 \mathrm{ps}$ and the effect of this travel time will be less than the time resolution of the electronics. However, since $10 \mathrm{eV}$ represents the tail end of the energy distribution, the effect is not likely to be visible in a typical atom probe system, even for low grid voltages. A comparison of mass spectra observed from the OPoSAP detector for a variety of grid bias voltages is 
shown in Figure 3. In all three cases, the input to the detector was held at $-50 \mathrm{~V}$ to avoid detection of secondary electrons from ion impacts on the walls of the vacuum chamber. There is no significant difference in the mass resolution, as measured from the width of the $\mathrm{Al}^{2+}$ peak, which is dominated by a timing resolution of approximately $2 \mathrm{~ns}$. One clear difference, however, is the minor peak which precedes the main peak, which is present for negative grid bias but disappears when the grid is biassed positively with respect to the MCP input. This peak is obviously due to secondary electrons generated when ions strike the nickel grid itself. Since the grid is $2.5 \mathrm{~mm}$ in front of the detector, and even low energy electrons travel at very high speeds compared to ions, the flight time detected from these events is $0.4 \%$ below those of ions striking the detector, leading to a peak $0.23 \mathrm{u}$ below the $\mathrm{Al}^{2+}$ peak.

A similar calculation can be performed to see how far a secondary electron might travel from the point of primary ion impact to the point of detection. The greatest distance will be traversed by electrons emitted at $45^{\circ}$ to the channel plate surface. In time $t$, an electron would travel a distance $x$ parallel to the channel plate surface, given by

$$
x=\frac{1}{\sqrt{2}} \sqrt{\frac{2 E}{m}} t=\sqrt{\frac{E}{m}} \frac{2 d}{e V} \sqrt{m E}=2 d \frac{E}{e V}
$$

Again using $E=10 \mathrm{eV}, V=10 \mathrm{~V}$ and $d=2.5 \mathrm{~mm}$, the distance travelled by the secondary electron would be $5 \mathrm{~mm}$, which would lead to a very significant degradation of the spatial resolution. Increasing the grid voltage to $200 \mathrm{~V}$ reduces the travel distance to $0.25 \mathrm{~mm}$. Since $10 \mathrm{eV}$ represents the maximum secondary electron energy, the actual spatial resolution will be somewhat better than this figure. The OPoSAP has a spatial resolution of approximately $0.25 \mathrm{~mm}$, and no degradation has been observed with grid bias as low as $15 \mathrm{~V}$. A more detailed analysis of the trajectory of photoelectrons has been performed by Taylor et al. [9], who considered the effect on detector resolution in a UV imaging system, and their results were similar to those given here.
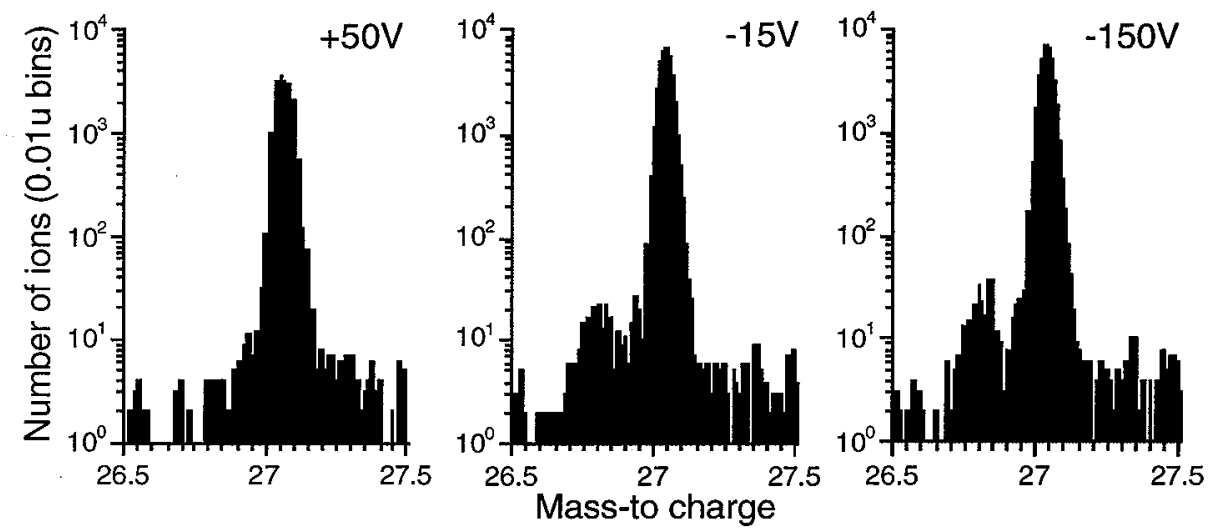

Figure 3: Comparison of mass spectra for $\mathrm{Al}^{2+}$ ions in the energy compensated optical PoSAP system, for different grid bias. The grid potentials given in each case are quoted with respect to the front face of the MCP, which was set to $-50 \mathrm{~V}$.

\section{DISCUSSION}

It is interesting to note that the results of Deconihout $e t$ al. on the variation of efficiency with grid voltage [4] differ from those reported here and by Funsten et al. [5] in two respects: the voltage required to achieve maximum eficiency and the efficiency decrease at high voltages. Deconihout $e t$ al. required $50 \mathrm{~V}$ before reaching peak efficiency, whereas we find a voltage of $5 \mathrm{~V}$ is sufficient, and Funsten $e t$ al. suggest an even lower voltage. Deconihout et al. used a negative bias on the front face of the MCP, so that the anodes in the detector were at ground potential. The negative bias generated a standing field with respect to the earthed surroundings, and a significant grid bias was required before this field was overcome and a net positive field existed at the channel plate surface. Results of electrostatic modelling given in Figure 4 shows the effect clearly. With the MCP input at $-2000 \mathrm{~V}$ (typical of that used by Deconihout $e$ t al.) and a grid potential of $-2020 \mathrm{~V}$ (Figure $4 \mathrm{~b}$ ), equipotentials at $-2005 \mathrm{~V}$ are restricted mainly to the space close to the grid wires. Most of the secondary electrons from the channel plate surface can escape past these regions and towards to regions at ground potential beyond. Only by raising the grid bias to higher values (Figure 4c) are the secondary electrons recovered from the whole of the area beneath the grid. 
a)
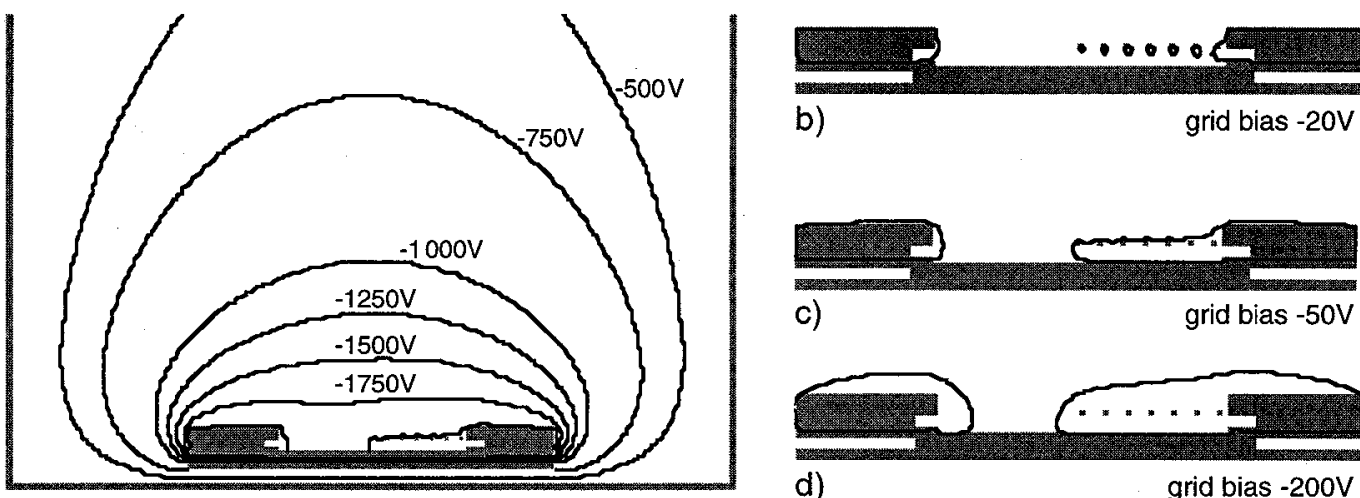

b)

grid bias $-20 \mathrm{~V}$

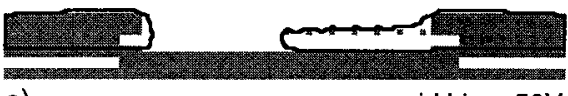

c)

grid bias $-50 \mathrm{~V}$

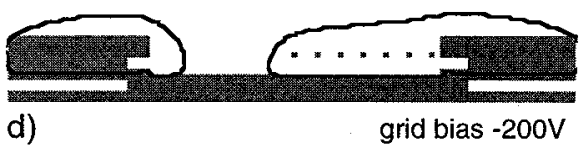

Figure 4: Results of 2-dimensional electrostatic modelling, to calculate the approximate potentials around the detector arrangement used by Deconihout et al. (a) Overall distribution of field, showing equipotentials at $250 \mathrm{~V}$ intervals. (b) Close up of the MCP surface $(-2000 \mathrm{~V})$ and grid $(-2020 \mathrm{~V})$ showing equipotential at $-2005 \mathrm{~V}$ which would be 'opaque' to secondary electrons generated at the surface with energies of less than $5 \mathrm{eV}$. (c) With a grid potential of $-2050 \mathrm{~V}$, the majority of the surface under the grid is 'covered' by the $-2005 \mathrm{~V}$ equipotential, so the maximum detection efficiency is obtained. (d) Raising the grid bias to $-2200 \mathrm{~V}$ leads to significant field spillage from the grid support, even over areas of the MCP surface not covered by the grid. This leads to an increase in efficiency in the uncovered area which makes it appear that the detection efficiency is decreasing in the area covered by the grid.

Raising the grid bias even further leads to field spillage from the space between the grid support and that of the MCP (Figure 4d), such that a region of positive field exists over the MCP surface which is not covered by the grid. Within this region, secondary electrons will be returned to the MCP surface and may be detected, just as they are below the grid. Deconihout et al. used the ratio of detection rates in the two regions to calculate detection efficiency, assuming the efficiency for the uncovered area was equal to $60 \%$. Thus an increase of detection efficiency within this 'control' region of the detector, due to fringing fields, would make it appear as if the detection efficiency in the region under the grid had decreased. An exact calculation of the effect would require a full 3-dimensional field calculation, but Figure 5 shows the results of an approximate calculation, using the 2-dimensional electrostatic modelling used here. It has been assumed that secondary electrons will be recovered wherever the surface field on the MCP is greater than 1 $\mathrm{V} / \mathrm{mm}$, yielding an efficiency of $90 \%$. Where the field is negative, the detection efficiency is assumed to equal the open area ratio of $60 \%$. Obviously the field will vary across the surface of the MCP covered by the grid, because of field spillage through the holes in the grid, but for the purposes of the calculation an average local field is used. Despite the simplicity of the calculations, the results follow the same general trend as the measurements of Deconihout $e t$ al., particularly in the rate of fall-off of detection efficiency with higher grid bias.

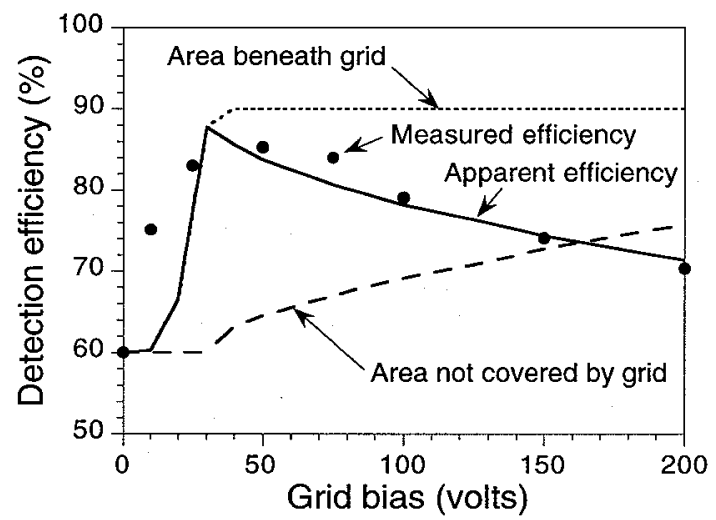

Figure 5: Variation in efficiency over different regions of the test detector used by Deconihout et al. and the overall efficiency which would have been measured by comparing the two and assuming an open area of $60 \%$. The efficiency data from Deconihout $e t$ $a l$. are shown for reference (points). 
Taylor et al. [9] observed a drop off in the detection efficiency for incident photons at large grid bias, when the photoelectrons generated from the interchannel web failed to reach the nearest channel, and are returned instead to the web. However, this effect was only appreciable for larger applied fields than are applied here (up $1000 \mathrm{~V} / \mathrm{mm}$ ), or for lower energy photoelectrons. Given the results of our electrostatic modelling, and calculations of the distance travelled by the secondary electrons (above) the decrease in detection efficiency observed by Deconihout et al. appears to be due instead to field fringing effects. This is confirmed by comparing pulse height distributions from the area of the channel plate under the grid, and the uncovered area, as shown in Figure 6. Charge distributions from the area under the grid (Figure 6a) show a broadening for low applied grid bias [2], due to the detection of secondary electrons. At higher fields, the distribution remains constant, whereas it would be expected to become narrower if the secondary electrons were no longer being detected. Conversely, the distributions from the uncovered area start to broaden at high fields, Figure 6(b), consistent with the detection of secondary electrons from this part of the MCP.

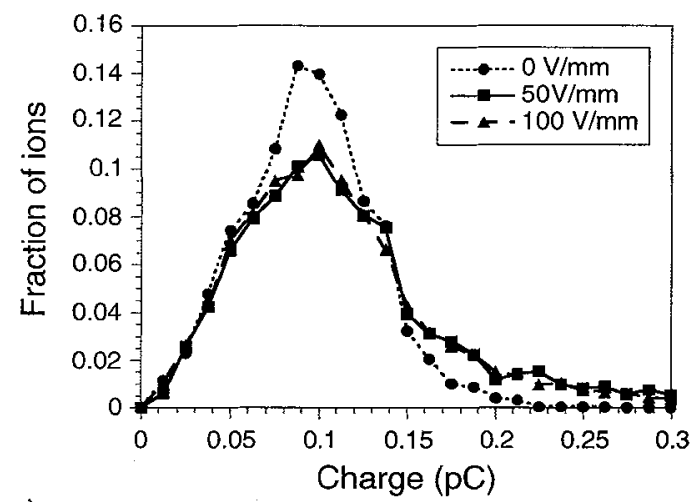

a)

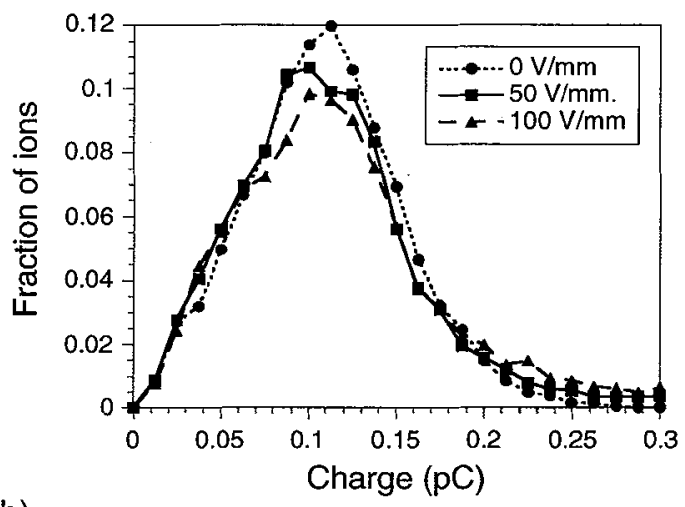

b)

Figure 6: Pulse height distributions from the work of Deconihout et al., [4]: (a) from the area of the MCP covered by the grid; (b) from the uncovered area of the MCP.

\section{Acknowledgements}

The authors would like to thank Dr. H.E. Bishop for helpul discussions. SJS is grateful to the Engineering and Physical Sciences Research Council (EPSRC) and Kindbrisk Limited for financial support in the form of a CASE studentship. AC thanks The Royal Society and Wolfson College (Oxford) for support during the course of this work. Professors B. Cantor and D.G. Pettifor are gratefully acknowledged for the provision of laboratory facilities. This research was supported by The Paul Instrument Fund of The Royal Society, and by the EPSRC under grant number GR/H/38485.

\section{References}

[1] Wiza J.L., Nucl. Instr. Meth. 162 (1979) 587-601.

[2] Panitz J.A. and Foesch J.A., Rev. Sci. Instrum. 47 (1976) 44-49.

[3] Gao R.S., Gibner P.S., Newman J.H., Smith K.A. and Stebbings R.F., Rev. Sci. Instrum. 55 (1984) 1756-1759.

[4] Deconihout B., Gerard P., Bouet M. and Bostel A., Appl. Surf. Sci. 94/95 (1996) 422-427.

[5] Funsten H.O., Suszcynsky D.M., Harper R.W., Nordholt J.E. and Barraclough J., Rev. Sci Instrum. 67 (1996) 145-154.

[6] Cerezo A., Godfrey T.J. and Smith G.D.W., Rev. Sci. Instrum.59 (1988) 862-866.

[7] Benninghoven A., Rüdenauer F.G. and Werner H.W., Secondary ion mass spectrometry: Basic concepts, instrumental aspects, applications, and trends (J. Wiley \& Sons, New York, 1987) pp. 143-172, and references therein.

[8] Klein H.J., Z. Physik 188 (1965) 78-92.

[9] Taylor R.C., Hettrick M.C. and Malina R.F., Rev. Sci. Instrum. 54 (1983) 171-176. 